\title{
CONTRIBUIÇÃO DA TOMOGRAFIA COMPUTADORIZADA NO DIAGNOSTICO E NO ESTUDO POS-OPERATORIO DOS TUMORES SELARES
}

\author{
J. JORGE FACURE * \\ NUBOR O. FACURE **
}

Até o advento da tomografia computadorizada (T.C.), o estudo pós-operatório dos tumores selares se restringia ao exame clínico-neurológico e às dosagens hormonais, reservando-se os métodos neurorradiológicos para os casos nos quais a avaliação clínica evidenciasse evolução desfavorável.

É objetivo deste trabalho comentar os resultados da T.C. em 18 pacientes com tumores selares e sobretudo analisar a contribuição da metodologia na avaliação tardia do resultado cirúrgico.

\section{CASUISTICA E METODOS}

Nossa casuística compreende 18 pacientes com tumores da região selar: 9 adenomas cromófobos, 5 craniofaringeomas, 2 colesteatomas, 1 adenoma eosinófilo e 1 glioma de quiasma (tabela 1). Em 14 deles realizamos craniotomia para exerese tumoral, e utilizamos em 4 a via trans-esfenoidal. Onze pacientes foram submetidos a microneurocirurgia e 7 a neurocirurgia convencional. Em 6 doentes realizamos tomografia computadorizada (T.C.) pré-operatória e em 12 o exame foi utilizado para a avaliação tardia da cirurgia.

Estudo pré-operatorio - O exame clínico-neurológico confirmou alterações visiais em todos os casos, exceto em um (caso 13). O exame de fundo de olho resultou normal em 3 pacientes (casos 11, 13 e 17), mostrou atrofia primária de papila bilateral em 10 (casos 1, 4, 5, 6, 7, 8, 10, 14, 16 e 18), atrofia primária unilateral em 4 (casos 2, 8, 12 e 15) e edema de papila em um (caso 3). O estudo do campo visual fol realizado em 8 doentes: confirmou hemianopsia bitemporal em 4 (casos 1, 8, 14 e 16); hemianopsia temporal em 2 (casos 2 e 9) e quadrantopsia superior em 2 (casos 6 e 10).

Alterações endócrinas foram encontradas em 11 pacientes: acromegalia no caso 13, ausência de caracteres sexuais secundários no caso 1, amenorréia e políría no caso 8: amenorréia e obesidade no caso 10 e apenas amenorréia no caso 5 ; perda do libido e ausência de caracteres sexuais secundários em 3 (casos 2,6 e 9) e nanismo nos casos 3,7 e 15.

Departamento de Neurologia e Neurocirurgia da Faculdade de Ciências Médicas da Universidade Estadual de Campinas.

"Professor assistente; "* Coordenador do Departemento. 


\begin{tabular}{|c|c|c|c|c|c|c|}
\hline Cor & Data & Tumor & Caso & Nome & Idade & Sexn \\
\hline $\mathbf{B}$ & 03-07-71 & Adenoma cromofobo & 1 & WP & 14 & $\mathbf{M}$ \\
\hline $\mathbf{B}$ & $12-10-72$ & Adenoma cromofobo & 2 & NH & 37 & $\mathbf{M}$ \\
\hline B & 24-05-73 & Craniofaringeoma & 3 & $\mathbf{R S}$ & 7 & $\mathbf{F}$ \\
\hline B & $28-09-73$ & Colesteatoma & 4 & NBC & 11 & $\mathbf{M}$ \\
\hline $\mathbf{B}$ & $18-09-75$ & Adenoma cromofobo & $\mathbf{5}$ & ASG & 53 & $\mathbf{F}$ \\
\hline $\mathbf{B}$ & $23-10-75$ & Adenoma cromofobo & 6 & CWP & 31 & $\mathbf{M}$ \\
\hline $\mathbf{B}$ & $03-05-76$ & Craniofaringeoma & 7 & JN & 8 & $\mathbf{M}$ \\
\hline B & $23-08-76$ & Adenoma cromofobo & 8 & $\mathrm{DQ}$ & 48 & $\mathbf{F}$ \\
\hline B & $08-09-76$ & Adenoma cromofobo & 9 & JC & 51 & $\mathbf{M}$ \\
\hline B & 29-09-77 & Craniofaringeoma & 10 & MSTC & 24 & $\mathbf{F}$ \\
\hline $\mathbf{P}$ & $20-10-77$ & Adenoma cromofobo & 11 & wC & 45 & $\mathbf{F}$ \\
\hline B & 21-12-77 & Adenoma cromofobo & 12 & EAS & 77 & $\mathbf{F}$ \\
\hline B & $31-01-78$ & Adenoma eosinofilo & 13 & NAT & 42 & $\mathbf{F}$ \\
\hline B & $18-05-78$ & Craniofaringeoma & 14 & ZM & 47 & $\mathbf{M}$ \\
\hline B & $09-06-78$ & Craniofaringeoma & 15 & MHP & 18 & $\mathbf{F}$ \\
\hline $\mathbf{B}$ & $25-10-78$ & Adenoma cromofobo & 16 & QS & 54 & $\mathbf{M}$ \\
\hline B & $04-01-80$ & Colesteatoma & 17 & ACRS & 27 & $\mathbf{F}$ \\
\hline B & $24-05-80$ & Glioma do quiasma & 18 & DMS & 26 & $\mathbf{F}$ \\
\hline
\end{tabular}

Tabela 1 - Dados de identificacão, idade em anos e data da cirurgia.

Hipertensão intracraniana foi evidenciada em 4 pacientes (casos 3, 7, 8 e 10).

A radiografia simples do crânio trouxe grande contribuição à avaliação pré-operatória. O exame mostrou alterações da sela turca em todos os casos exceto em um (caso 14). A alteração mais encontrada fol o aumento do volume selar com erosão do dorso e assoalho da sela (casos 1, 4, 6, 8, 9, 12, 16, 17, 18). Em dois casos (casos 5 e 10) havia apenas erosão do dorso selar e em outros dois (casos 11 e 13) apenas aumento do volume selar. Calcificação selar e supra-selar foram diagnosticadas em 2 pacientes (casos 3 e 4). Para estes pacientes o estudo radiológico além das alterações descritas evidenciou sela turca em jota nos casos 3 e 4 e hipertensão intra-craniana nos casos 3 e 7.

O estudo angiográfico encefálico foi realizado em todos os pacientes exceto em 2 (casos 1 e 2). A angiografia cerebral carotídea direita foi realizada em 10 pacientes (casos 3, 4, 5, 6, 10, 11, 12, 13, 16 e 17). Para estes casos, com a técnica de compressão da carótida esquerda, conseguimos contrastar o segmento A1 de ambas artérias cerebrais anteriores. O exame resultou normal em 3 pacientes (casos 10, 13 e 17), confirmando a neoplasia selar com expansão supra-selar nos demais. Realizamos angiografia carotídea bilateral em dois pacientes (casos 9 e 18) que confirmou expansão supra-selar do tumor. A angiografia carotidea direita e angiografia da vertebral diagnosticou a expansão supra-selar em 2 pacientes (casos 7 e 8 ). Foi realizado em 2 doentes (casos 14 e 15) estudo angiográfico encefálico completo por cateterismo femural: o exame resultou normal para ambos. 
Pneumocisternografia fol realizada em todos os pactentes exceto para os casos 8, 12 e 17. Fim todos os casos o exame mostrou expansão tumoral supra-selar.

A tomografia computadorizada cefálica fol realizada em 6 pacientes: resultou normal em um caso (caso 14) e confirmou a neoplasia nos outros 5 (casos 10, 12, 15, 17 e 18).

Cirurgia - Realizamos craniotomia frontal direita em 10 pacientes (casos 1, 2, 3. 4, 5, 6, 8, 9, 10, 14 e 18); craniotomia fronto-temporal direita em 4 (casos 3, 7, 15 e 16) - a vla transesfenoidal foi utilizada 4 vezes (casos 11, 12, 13 e 17). Onze pacientes foram submetidos a microneurocirurgia (casos 3, 4, 5, 8, 11, 12, 13, 15, 16, 17 e 18) e os 7 restantes a neurocirurgia convencional (casos 1, 2, 6, 7, 9, 10 e 14).

Estudo pós-operatorio - Para a avaliaça tardia da terapêutica cirúrgica realizamos tomografia computadorizada em 12 pacientes (casos 1, 2, 3, 4, 5, 6, 7, 8, 9, 11, 15 e 16). Em 5 pacientes o exame resultou normal (casos 2, 5, 9, 11 e 13). Em outros 5 confirmou recidiva (casos 1, 4, 6, 8 e 16). Hhm um doente o exame mostrou a presenca de dols clstos supra-selares (caso 7): y em outro (caso 3) fol diagnosticada dilataça ventricular.

Realizamos também angiografia cerebral em 5 pacientes. O exame resultou normaj em 2 (casos 1 e 11) e confirmou recidiva nos outros 3 (casos 4, 7 e 8).

Dols pacientes (casos 1 e 7) foram submetidos também a pneumocisternografia. 0 exame confirmou expansão supra-selar em ambos.

Radioterapia - Cinco pacientes de nossa casuistica (casos 2, 3, 11, 13 e 18) foram encaminhados para telecobaltoterapia tendo recebido cada um a dose de 5000 rads na regiăc selar.

Reoperagao - Dos 5 pacientes em que a tomografia computadorizada indicou recidiva do tumor, apenas 2 (casos 4 e 8) foram reoperados. Para o caso 4 a craniotomia confirmou a recidiva. Realizamos exerese total com técnica microneurocirúrgica. No caso 8 a craniotomia mostrou aderencias ao nivel do quiasma optico e tecido cicatricial intra-selar. Para um paciente (caso 7) em que a recidiva fol confirmada pela angiografia cerebral, realizamos craniotomia com esvaziamento de tumor tendo sido colocado o reservatório do Ommaya. Este mesmo paciente submeteu-se a outra reoperaçăo para esvardamento de dols cistos supra-selares identificados pela tomografia.

Em um paciente (caso 3) realizamos derivaço ventriculoperitoneal.

\section{RESULTADOS}

Para 12 pacientes de nossa casuística, o resultado p6s-operatório pode ser avaliado pela tomografia computadorizada. Para estes casos o periodo de seguimento até a realizacăo da T.C. variou de 2 a 80 meses (tabela 2) o exame resultou normal em 5 pacientes (casos 2, 5, 9, 11 e 13) em outros 5 fol confirmada recidiva (casos 1, 4, 6, 8 ( 16). Destes, 2 foram reoperados (casos 4 e 8) apresentando boa evolucão pós-operatória com seguimento de 5 e 7 meses respectivamente, após a reoperaçăo. Em um pactente (caso 3) o exame mostrou acentuada dilatagaro ventricular, tendo aldo indicada 
derivação ventriculoperitoneal (D.V.P.). A evolução clínica foi satisfatória e o período de seguimento após a D.V.P. fol de 12 meses. Para outro paciente (caso 7) a tomografia computadorizada confirmou a presença de 2 clstos supra-selares que foram esvaziados. Houve evoluçăo clínica satisfatória e o perfodo de seguimento após a reoperação fol de 10 meses.

Em 6 pacientes não fol realizada a tomografia no pós-operatório. Destes, 2 faleceram (casos 10 e 12). Os demais (casos 14, 15, 17 e 18) apresentam atualmente evolução clínica satisfatória e foram acompanhados por 19, 5, 4 e 1 meses respectivamente.

\begin{tabular}{rlcl}
\hline Caso & Tumor & $\begin{array}{c}\text { Seguimento } \\
\text { em meses }\end{array}$ & Resultado da T.C. \\
\hline \hline 1 & Adenoma cromofobo & 80 & Recidiva \\
2 & Adenoma cromofobo & 64 & Normal \\
3 & Craniofaringeoma & 58 & Dilataça ventricular \\
4 & Colesteatoma & 57 & Recidiva \\
5 & Adenoma cromofobo & 31 & Normal \\
6 & Adenoma cromofobo & 29 & Recidiva \\
7 & Craniofaringeoma & 24 & Cisto supra-selar \\
8 & Adenoma cromofobo & 24 & Recidiva \\
9 & Adenoma cromofobo & 18 & Normal \\
11 & Adenoma cromofobo & 4 & Normal \\
13 & Adenoma eosinofilo & 7 & Normal \\
16 & Adenoma cromofobo & 7 & Recidiva \\
\hline
\end{tabular}

Tabela \& - Avaliaçó pos-operatória pela tomografia computadorieada.

Complicagoes infecciosas - Diagnosticamos meningite no pós-operatório imediato em três pacientes (casos 6,12 e 13) e infecçáo no retalho cirágico no caso 9. Destes pacientes, nos casos 12 e 13 foi utilizada a via trans-esfenoidal. Um dos pacientes faleceu (caso 12) e os outros apresentaram boa evoluçáo clínica com o uso de antibióticos. Para o caso 9 tivemos que retirar o retalho bsseo para cura da infecçá.

Observamos fistula liquorica em 2 pacientes (casos 12 e 13), ambos apresentaram também meningite bacteriana.

Distúrbio hipotalamico - Fol verificado no pós-operatório de 4 pacientes portadores de craniofaringeomas (casos 3, 7, 10 e 14), em um paciente com colesteotoma (caso 4) e em 2 pacientes com adenomas (casos 1 e 6). Todos estes pacientes apresentaram diabetes insipido que foi facilmente controlado exceto no caso 10, que apresentou também hipertermia e convulsão evoluindo para o obito.

Causas do bbito - Dois pacientes de nossa casufstica faleceram (casos 10 e 12). 0 primeiro foi operado de urgência devido a hipertensão intracraniana severa. A cirurgia consistiu em exerese total de um craniofaringeoma cistico. No pós-operatório imediato foram diagnosticados atelectasia pulmonar e distúrbio hipotalamico. O óbito ocorreu na segunda semana do p6s-operatório. Para o caso 12, uma paciente submetída a exerese de um adenoma cromófobo por via trans-esfenoidal, o obito fol atribuido a fistula liquórica, meningite bacteriana e broncopneumonia. 


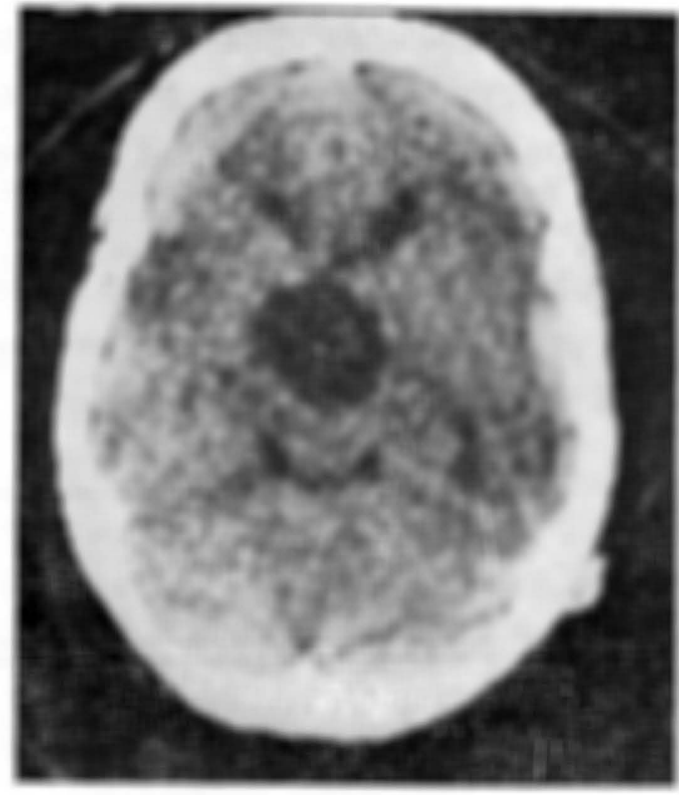

Fig. 1 - Caso 10, craniofaringeoma

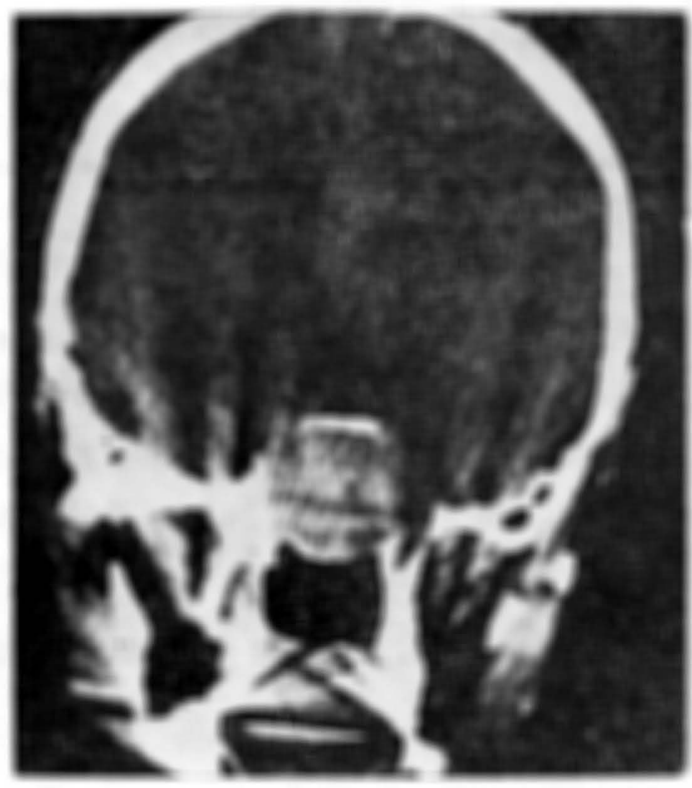

Fig. 3 - Caso 17, colesteatoma

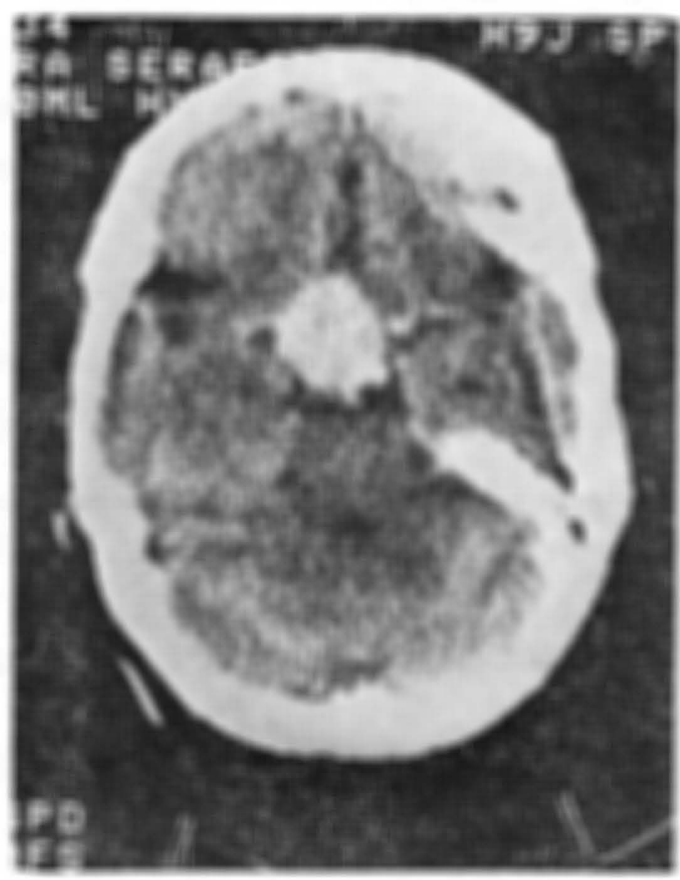

Fig: 2 - Caso 12, adenona cromofobo

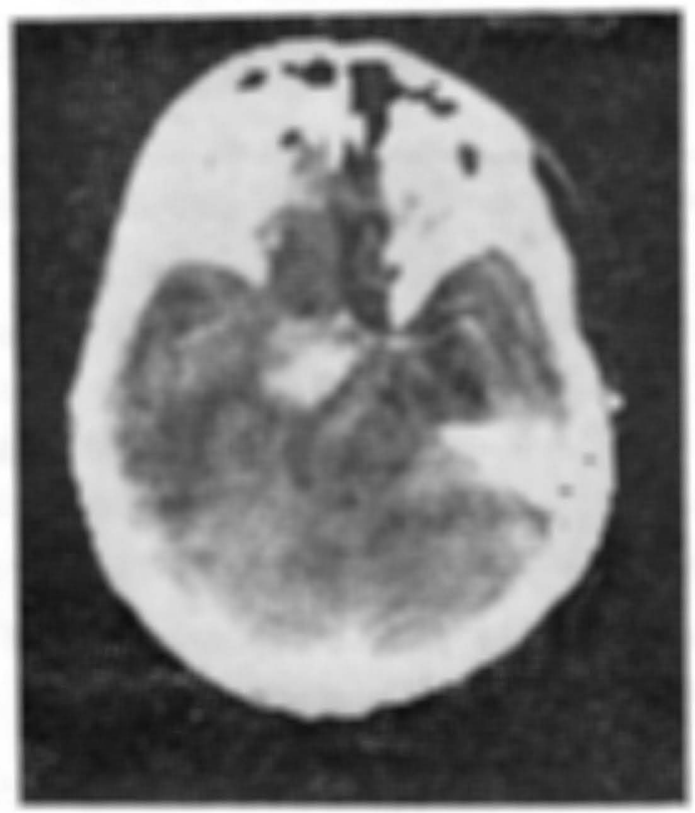

Wrig. 4-Caso 18, glioma do quiasma

\section{COMENTARIOS}

A tomografia computadorizada (T.C.) tem sido recomendada como exame inicial para a investigação dos tumores da região selar 6,7,8. Os primeiros trahalhos puhlicados sobre esta metodologia referem que a alta densidade das estruturas ósseas adjacentes à sela turca e a baixa densidade do ar no seio esfenoidal produzem artefatos que dificultam a visualização do tumor 1,2,4. 
Ambrose e col. 2 realizaram T.C. em 366 pacientes com tumores intracranianos. Em 16 casos a tomografia resultou normal sendo que em 8 o tumor era da região selar. Em um paciente de nossa casuística (caso 14) a T.C. resultou normal. $\mathrm{O}$ diagnóstico foi feito pela pneumocisternografia fracionada, tendo a cirurgia confirmado tratar-se de craniofaringeoma cístico.

Leeds e Naidich 5 , esquematizaram as alterações encontradas na T.C. de 84 pacientes com lesões selares. Iniciam o exame com um corte passando pelo seio esfenoidal com $13 \mathrm{~mm}$ de espessura e continuam a tomografia através da sela turca, cisterna supra-selar e porção anterior do III ventrículo com intervalos de $10 \mathrm{~mm}$. O exame é repetido após a injeção intravenosa de contraste (diatrizoato de meglumine). Com este procedimento referem que apenas os microadenomas não são diagnosticados pela T.C. Para os adenomas da hipofise o exame permite demonstrar o conteúdo intra-selar bem como a sua extensão extra-selar. Também pode ser avaliado o alargamento selar, a erosão dos processos clíonoideos e do assoalho da sela turca. O exame poderá demonstrar. também dilatação das cisternas pré-pontina e inter-penducular quando há expansão supra-selar do tumor. Os autores encontraram em 2 pacientes desta série calcificações supra-selares semelhantes às encontradas nos craniofaringeomas. Esta ocorrência torna difícil o diagnóstico diferencial pela T.C. entre os dois tumores. Para os craniofaringeomas as alterações mais comumente encontradas são: calcificações, lesão supra-selar cística e hidrocefalia.

Reich e col 9, apresentaram 20 pacientes com lesões justa-selares diagnosticadas pela T.C. Foram incluídos na casuística um aneurisma da carótida e um aneurisma da artéria basilar. O menor tumor demonstrado media $10 \mathrm{~mm}$. As lesões foram diagnosticadas pela angiografia cerebral em $88 \%$ dos casos e pela T.C. em $95 \%$ dos casos.

Para os 6 pacientes de nossa casuistica nos quais a tomografia foi feita no pré-operatório, o exame resultou normal em 1 (caso 14). A natureza da neoplasia sugerida pela T.C. foi confirmada em 3 pacientes (casos 10, 12 e 15), para o caso $17 \mathrm{em}$ que a T.C. sugeriu tratar-se de adenoma o estudo histopatológico confirmou colesteatoma. Para o caso 18 a T.C. mostrou uma pequena massa tumoral intra-selar com expansão volumosa supra selar com maior volume à esquerda da sela turca. A cirurgia revelou tratar-se de glioma do quiasma óptico (Figuras dos casos 10, 12, 17 e 18).

Ezrin ${ }^{3}$ recomenda que a T.C. para o estudo da região selar e das cisternas supra-selares deve ser iniciada por um corte que passa pela sela turca em um plano paralelo à linha de base de Reid (linha órbito-meatal) os cortes realizados antes e após a injeção endovenosa de contraste devem ser idênticos, permitindo um estudo comparativo. Desta forma, analisando a retenção do contraste intra-selar o autor refere ser possivel diagnosticar micro-adenomas de 5 a $7 \mathrm{~mm}$.

Leeds e Naidich 5 salientam que as alterações determinadas pelo manuseio cirúrgico dos tecidos podem quando avaliadas tardiamente pela T.C. deixar dúvidas quanto a ocorrência de recidiva do tumor. 
Dos 12 pacientes de nossa casuística em que realizamos T.C. no pós-operatório o exame sugeriu recidiva em 5. Apenas 2 foram reoperados. Em 1 páciente (caso 4) o estudo angiográfico revelou expansão supra-selar e a cirurgia confirmou a recidiva. No outro paciente (caso 8) em que a angiografia e a T.C. sugeriram recidiva, a reoperação mostrou apenas aderência ao nivel do quiasma e tecidos cicatricial intra-selar. Dos outros 3 pacientes em que a T.C. sugeriu recidiva 2 (casos 6 e 16) não aceitaram novos exames. Para o outro paciente (caso 1) a angiografia carotídea resultou normal, a pneumocisternografia fracionada mostrou expansão supra-selar mas o paciente não aceitou ser reoperado.

\section{RESUMO}

E analisada casuística de 18 pacientes com tumores da região selar com o uso da tomografia computadorizada (T.C.). Em 6 deles o exame foi feito antes da cirurgia e confirmou a neoplasia em 5 , sendo que a natureza do tumor foi sugerida corretamente em 3 casos. Para 12 doentes a T.C. foi feita para estudo tardio do resultado cirúrgico. Resultou normal em 5 casos, mostrou a existência de um cisto supra-selar em 1, dilatação ventricular em outro e sugeriu recidiva nos 5 restantes. Dos 5 pacientes em que a T.C. sugeriu recidiva, dois foram reoperados. Em um paciente com colesteatoma a cirurgia confirmou a recidiva e para o outro, com adenoma cromófobo a reoperação mostrou apenas tecido cicatricial e aderências na região selar.

\section{SUMMARY}

Computerized tomography in the diagnosis and follow-up of sellar tumors.

The computerized tomography (C.T.) of 18 pacients with sellar tumours were analysed. The C.T. made before surgery in 6 cases was positive in 3 and the type of tumour suggested by C.T. was confirmed in 3. Twelve pacients had a C.T. investigation after surgery and the examination suggest recurrent tumour in 5. Two of these were re-operated. One pacient with colesteatoma had the recurrent tumour verified by surgery and the other, with a pituitary adenoma during re-operation was noted only cicatricial tissue at sellar region.

\section{REFERENCIAS}

1. AMBROSE, J. Computerized x-ray scanning of the brain. J. Neurosurg. 40:679, 1974

2. AMBROSE, J.; GOODING, M. R. \& RICHARDSON, A. E. - An assessment of the accuracy of computerized transverse axial scanning (EMI Scanner) in the diagnosis of intracranial tumours. Brain, 98:569, 1975.

3. EZRIN, C. - Clinícal pituitary disorders. Bull, Lus Angeles Neurol. Soc. 42:81, 1977. 
4. GAWLER, J.; Du BOULAY, G. H.; BULL, J. W. D. \& MARSHALL, J. - Computer-assisted tomography: its place in investlgation of sispected intracranial tumours. Lancet II: 419, 1974.

5. LEEDS, N. E. \& NAIDICH, T. P. - Computerized tomography in the diagnosis of sellar and parasellar lesions. Semin. Roentgenol. 12:121, 1977.

6. NAIDICH, T. P.; PINTO, R. S.; KUSHNER, M. J.; LIN, J. P.; KRICHEFF I. I.: LEEDS, N. E. \& CHASE, N. E. - Evaluation of sellar and parasellar masses by computed tomography. Radiology, 120:91. 1976.

7. NEW, P. F. J.; SCOTT, W. R.; SCHNUR. J. A.: DAVIS, K. R. \& TAVERAS, J. M. - Computerized axial tomography with the EMI Scanner. Radiology 110:109, 1974.

8. POST, M. J. D.; DAVID, N. J.; GLASER, J. S. \& SAFRAN, A. - Pituitary apoplexy: diagnosis by computed tomography. Radiology, 134:665, 1980.

9. REICH, N. E.; ZELCH, J. V.; ALFIDI, R. J.; MEANEY .T. F.: DUCHESNEAU, P. M. \& Whinstein, M. A. - Computed tomography in the detection of juxtasellar lesions. Radiology, 118:333, 1976.

Clinica Neurol6gica - Fraculdade de Ciencias MÉdicas - Universidade Estadual do Campinas - Caixa Postal 1170 - 13100 Campinas, SP - Brasil. 